\title{
Aspectos metodológicos da prescrição da caminhada e a questão dose-resposta na prevenção de doenças
}

\author{
Methodological aspects of walking prescription and dose-response \\ relationship in disease prevention
}

Raul A. Freire

Brenno S. Silva

Walace D. Monteiro*

\section{Resumo}

O sedentarismo é um dos maiores problemas de saúde pública no Brasil e no mundo, que leva a uma grande incidência de várias doenças e mortalidade por diversas causas. Em contrapartida, a prática regular de exercícios traz diversos benefícios à saúde. Dentre as várias formas de atividade física, a caminhada é uma das mais praticadas pela população devido à facilidade para realização, ao baixo custo e ao reduzido risco de lesões. Adicionalmente, a literatura tem demonstrado que a caminhada pode ser bastante útil na prevenção e tratamento de diversas doenças, reduzindo ou estabilizando os sintomas e diminuindo a quantidade de medicamentos. No entanto, os dados referentes à questão dose-resposta para observar essas melhoras ainda são inconsistentes. Além disso, as diretrizes que tratam da progressão do treinamento envolvendo a caminhada ainda são muito gerais. O presente artigo

Revista HUPE, Rio de Janeiro, 2013;12(4):89-98 doi:10.12957/rhupe.2013.8716 trata especificamente desses temas e objetiva detalhar algumas questões relacionadas à doseresposta da prática da caminhada em diferentes problemas de saúde, bem como tecer algumas considerações metodológicas que envolvem a prescrição segura e eficiente desta atividade. Para tanto, o texto foi organizado em três tópicos. No primeiro, é abordada a questão dose-resposta da prática da caminhada e seus benefícios à saúde. $\mathrm{O}$ segundo tópico abordado diz respeito à atuação específica da caminhada na prevenção e tratamento de algumas doenças e fatores de risco, bem como na redução no uso de medicamentos. No terceiro tópico, são tecidas algumas considerações metodológicas para prescrição da caminhada, introduzido neste contexto o papel da detecção da velocidade de transição caminhada-corrida para prescrição do exercício.

Descritores: Caminhada; Exercício físico; Treinamento; Prevenção de doenças; Saúde. 


\section{Abstract}

The sedentariness is one of the major public health problems in the world, leading to an increase of disease incidences and mortality due to several cases. Nevertheless, the regular practice of exercise leads to several benefits to health. Among the ways of physical activity, walking is one of the most performed by the population because it is easier to be executed and implies low cost and reduced risk of injuries. In addition, literature has demonstrated that walking may be quite useful in prevention and treatment of several diseases, reducing or stabilizing the amount of medicines. However, the data regarding the dose-response relationship in order to observe these improvements are still inconsistent. Besides, training guidelines involving the walking are too much general as well. This study investigated especially these subjects and aims to detail some questions associated with the dose-response relationship of the practice of walking in different health problems, as well as discussing some methodological considerations that involve efficient and safe prescription of this activity. Thus, the text was divided up into three topics. First, the doseresponse relationship regarding the practice of walking was discussed. The next topic addressed the function of walking in prevention and treatment of some diseases and risk factors, as the reduction of the medication use. Lastly, methodological considerations with respect to walking prescription were discussed along with the role of detection of walk-run transition to exercise prescription.

Keywords: Walking; Exercise; Training; Disease prevention; Health.

\section{Introdução}

A inatividade física tem sido considerada um problema de saúde pública, devido à maior incidência de doenças a ela relacionada, bem como ao consequente aumento dos custos no sistema de saúde. Quando associada ao consumo excessivo de bebidas alcóolicas, tabaco e alimentação inadequada, as consequências negativas para a saúde tornam-se ainda maiores. ${ }^{1}$

Dados recentes apontam que $31 \%$ das pessoas em todo o mundo não atendem à quantidade mínima de atividade física que deveria ser realizada, o que representa um problema de saúde pública. ${ }^{2}$ Se por um lado a inatividade física tem sido apontada como um grave problema, a prática regular de exercícios tem sido recomendada como estratégia não farmacológica de prevenção e tratamento de doenças e sintomas relacionados. ${ }^{3-5}$

Dentre as diversas modalidades de exercício físico, destacamos a caminhada, por ser uma atividade democrática em relação à adesão e acesso aos locais de treinamento, bem como ao baixo custo. ${ }^{6}$ Além disso, trata-se de uma atividade que não exige técnica para a sua realização, apresenta baixo nível de lesão e pode ser realizada por grandes massas populacionais. $^{7-9}$

Em recente trabalho enfocando o papel da caminhada, foi lançada a seguinte questão: pode uma atividade tão simples ser de grande importância para a saúde pública? A resposta foi sim. De fato, diversos estudos têm demonstrado que a caminhada pode atuar na prevenção e tratamento de doenças. Contudo, para que efeitos positivos possam ser alcançados é necessário entender algumas considerações metodológicas que envolvem a prescrição da caminhada. Desta forma, o objetivo desta revisão é detalhar algumas questões relacionadas à doseresposta da prática da caminhada em diferentes problemas de saúde, bem como tecer algumas considerações metodológicas que envolvem a prescrição segura e eficiente desta atividade.

Quanto de caminhada é necessário?

Até 1990 a prescrição do exercício era mais focada nas atividades vigorosas, com o objetivo de aumentar a aptidão física. A partir daí as atividades moderadas, como a caminhada rápida, começaram a ser prescritas para a promoção da saúde como alternativa para os indivíduos com menor aptidão física. $\mathrm{O}$ 
American College of Sports Medicine ${ }^{10}$ publicou algumas diretrizes de prescrição do exercício para indivíduos que desejam melhorar a aptidão física e saúde, as quais incluem intensidade, duração e frequência semanal de treinamento. Neste sentido, as atividades de intensidade moderadas são mais preconizadas, devendo ser realizadas cinco vezes por semana durante 30 60 minutos diários. Em alguns casos, atividades mais intensas podem ser realizadas durante um tempo mínimo de 20 minutos, ao menos três vezes por semana.

Apesar dessas recomendações gerais, surge a seguinte questão: qual a dose-resposta necessária para promover os benefícios relacionados à caminhada? Alguns estudos tentaram esclarecer tal questão e um mínimo de $1.000 \mathrm{kcal}$ por semana tem sido preconizado. Esse volume de treinamento corresponde em torno de 150 minutos de atividades moderadas e 75 minutos de atividades intensas por semana, $o$ que estaria associado a menores taxas de doença cardiovascular e morte prematura. ${ }^{10}$

Um estudo conduzido por Landi e colaboradores ${ }^{11}$ verificou a relação entre caminhada regular e risco de morte por todas as causas. Os autores acompanharam 248 idosos de ambos os sexos com independência funcional nas atividades diárias por 24 meses. O acompanhamento do volume de treinamento foi realizado por questionário e os indivíduos deveriam se enquadrar em quatro categorias, a saber: caminhavam 30 minutos ou menos; 30 minutos; 60 minutos ou mais que 60 minutos. A partir das respostas obtidas, o grupo foi dividido entre os que caminhavam menos e mais que 60 minutos para análise estatística. Dentre os resultados encontrados, destacamos que o grupo que caminhava 60 minutos ou mais, que teve menor probabilidade de depressão e uma menor prevalência de deficit cognitivo, insuficiência cardíaca congestiva, hipertensão e artrose, além de menor uso de remédios. Em relação à probabilidade de morte, após o ajuste para idade, sexo e outros potenciais fatores intervenientes, o grupo que caminhava uma hora ou mais teve menos chance de morrer quando comparado ao grupo sedentário. Apesar de limitado controle das variáveis de prescrição do exercício (intensidade, duração e frequência), a caminhada, enquanto atividade moderada, se mostrou benéfica para a promoção da saúde e redução do risco de morte nesses indivíduos.

Em estudo similar, Nagai e colaboradores ${ }^{12}$ investigaram 27.738 indivíduos de ambos os sexos com idades entre 40 e 79 anos, por um período de 13 anos. O objetivo foi examinar a associação entre o tempo gasto com a caminhada diária, a expectativa de vida e o tempo de vida gasto com tratamento médico. Os resultados mostraram que o grupo que caminhou mais de 1 hora por dia teve maior expectativa de vida aos 40 anos e menor gasto com tratamento médico do que o grupo que caminhou menos de 1 hora por dia. Apesar deste estudo não ter controlado a intensidade do esforço realizado pelos sujeitos, seus resultados demonstraram que a duração pode ter compensado em parte a intensidade baixa, já que os sujeitos foram instruídos a caminhar na velocidade de conforto.

No que diz respeito ao uso de medicamentos, a questão dose-resposta da caminhada também tem sido investigada. Em um dos experimentos, Willians ${ }^{13}$ verificou a relação entre quantidade, frequência e intensidade da caminhada com a utilização de medicamentos anti-hipertensivos, hipoglicemiantes e hipolipemiantes. Como principais resultados, verificou-se que os homens e mulheres que caminhavam a uma velocidade de $2,1 \mathrm{~m} / \mathrm{s}$ obtiveram $69 \%$ e $59 \%$, respectivamente, menos chance de usar remédios hipoglicemiantes do que aqueles que caminhavam a uma intensidade de $1,2 \mathrm{~m} / \mathrm{s}$. Relações semelhantes e significativas também foram encontradas entre distância semanal percorrida ( $\mathrm{km} /$ semana) e maior distância percorrida em um dia de caminhada e redução de anti-hipertensivos, hipolipemiantes e hipoglicemiantes.

Apesar dos benefícios proporcionados pela prática da atividade física, a questão doseresposta associada aos efeitos da caminhada deve 
ser analisada com cautela, já que, indivíduos com diferentes necessidades requerem uma prescrição individualizada quanto ao volume e intensidade do esforço ao se exercitarem. Além disso, uma mesma velocidade que caracteriza a caminhada como forma ideal de locomoção para um indivíduo, pode ser usada como corrida para outro. Por isso, as estratégias de prescrição do exercício devem levar em conta diversos aspectos que incluem ao menos o estado clínico do sujeito e sua condição física. O somatório desses aspectos deve ser analisado com cautela para se estabelecer a dose-resposta associada à prescrição da caminhada. Tal aspecto será detalhado em seção posterior, destinada aos aspectos metodológicos da prescrição da caminhada.

\section{O papel da caminhada na prevenção e tratamento de doenças}

As doenças cardiovasculares (DCVs) estão entre as principais causadoras de morte no mundo. Desde 1900, as DCVs são as principais causas de morte nos Estados Unidos. Estima-se que 83,6 milhões de americanos acima dos 20 anos tenham pelo menos um tipo de doença cardiovascular. Somente em 2009, 32,3\% das mortes foram ocasionadas por doença cardiovascular, gerando um custo anual de 312 , 6 bilhões de dólares. ${ }^{5}$ Já no Brasil, os dados mais atualizados do DATASUS estimam que 96.386 dos óbitos tenham sido por doença isquêmica do coração, o que representa $50 \%$ das mortes. ${ }^{14}$

Dentre as atividades físicas que podem ser usadas na prevenção e tratamento das DCVs a caminhada tem sido uma das mais preconizadas. Ainda que a caminhada possua uma intensidade mais reduzida em relação a outras atividades, como por exemplo, a corrida, existem evidências consistentes acerca de sua relação com a diminuição dos fatores de risco cardiovasculares. ${ }^{8,15,16}$ Outro aspecto positivo da caminhada diz respeito à facilidade para o controle da intensidade do esforço, aspecto fundamental para indivíduos mal condicionados ou mesmo aqueles que apresentam algum problema de saúde e que necessitam de maiores cuidados no controle da atividade.

No que diz respeito aos fatores de risco cardiovasculares, a caminhada apresenta resultados significativos na melhora de diversos indicadores fisiológicos relacionados ao risco de morte. Farinatti e colaboradores ${ }^{17}$ por exemplo, verificaram o efeito de um programa de caminhada de intensidade moderada (6080\% FCmáx), três vezes por semana durante 30 minutos, em indivíduos hipertensos. Os sujeitos apresentaram reduções significativas na relação cintura-quadril, percentual de gordura e pressão arterial, tanto sistólica, quanto diastólica $(-6 \mathrm{mmHg}$ e $-9 \mathrm{mmHg}$ respectivamente), o mesmo não ocorrendo com os seus pares que não se exercitaram. Liu e colaboradores ${ }^{18}$ corroboraram os resultados do estudo anterior ao realizarem um programa de exercício supervisionado em indivíduos pré-hipertensos. Os sujeitos caminharam por oito semanas a $65 \%$ do $\mathrm{VO}_{2}$ máx, quatro vezes por semana. Os resultados mostraram melhoras não só na pressão arterial sistólica e diastólica, mas também na modulação autonômica da frequência cardíaca e da sensibilidade barorreflexa.

Já para diabéticos a chance de uso de medicamento foi $64 \%$ menor para homens que caminhavam entre 5 e $15 \mathrm{~km} /$ semana. $^{13}$ Para esses pacientes a literatura também tem mostrado modificações importantes nos fatores de risco cardiovascular. Walker e colaboradores, ${ }^{19}$ por exemplo, observaram que 60 minutos de caminhada em velocidade autosselecionada, cinco vezes por semana, reduziram significativamente a hemoglobina glicada, o LDL colesterol e o colesterol total. Além disso, houve redução na gordura da região abdominal (medida através de densitometria de dupla captação - DEXA). Tal resultado é importante, visto que a gordura na região central é altamente relacionada a doenças do coração. ${ }^{20}$

No que se refere ao risco de morte, Smith 
e colaboradores ${ }^{16}$ verificaram que idosos diabéticos que caminhavam mais do que uma milha e meia por dia obtiveram redução aproximada de $50 \%$ de chance de morte por complicações cardiovasculares, do que os que não alcançavam essa distância diária. Uma das possíveis explicações para esse fenômeno pode estar na melhora do controle autonômico proporcionado pela caminhada. Nesse sentido, Figueroa e colaboradore ${ }^{21}$ verificaram aumento da sensibilidade barorreflexa e da variabilidade da frequência cardíaca pós-exercício em diabéticos obesos, depois de um programa de caminhada realizado quatro vezes por semana, durante 16 semanas, a uma intensidade de $65 \%$ do $\mathrm{VO}_{2}$ pico. Os mesmos resultados também foram encontrados em obesos não diabéticos, que compuseram o grupo-controle do experimento.

Com relação à obesidade, Bond Brill e colaboradores ${ }^{15}$ se preocuparam em investigar a importância do volume de treinamento sobre diversos fatores de risco em indivíduos com IMC acima de $30 \mathrm{~kg} / \mathrm{m}^{2}$. Para tanto, 56 indivíduos foram acompanhados por 12 semanas, após serem distribuídos aleatoriamente em três grupos, a saber: grupo dieta; grupo dieta mais caminhada de 30 minutos; e por fim o grupo dieta mais caminhada de 60 minutos. Todos os participantes se exercitaram cinco vezes por semana. Apesar de todos os grupos apresentarem mudanças positivas na pressão arterial, peso corporal e triglicerídeos, só os grupos caminhada demonstraram melhoras no LDL-colesterol, diâmetro sagital abdominal e circunferência abdominal, independentemente da duração do exercício. Esses achados ressaltam que a prática de 30 minutos de caminhada diária já seria suficiente para redução dos riscos cardiovasculares.

Outra doença com risco potencial para a saúde cardiovascular é a doença renal. ${ }^{22}$ Apesar da informação sobre os efeitos da caminhada nessa enfermidade ainda serem iniciais, alguns autores verificaram resultados positivos ao utilizarem a caminhada como tratamento não medicamentoso para essa enfermidade. Kosmadakis e colaboradores, ${ }^{23}$ por exemplo, verificaram o efeito de seis meses de caminhada na função renal, indicadores de risco cardiovascular e qualidade de vida de pacientes em estágio de pré-diálise. A realização de 30 minutos de caminhada diária, durante cinco semanas gerou melhoras significativas na qualidade de vida, composição corporal e manutenção da reatividade cardiovascular, o mesmo não ocorrendo no grupo-controle. Entretanto, o resultado mais interessante desse experimento recaiu na estabilização da medicação anti-hipertensiva no grupo exercício e aumento da mesma no grupocontrole, realçando a importância da caminhada como tratamento não medicamentoso nesses pacientes.

Outro estudo digno de nota foi conduzido por Mustata e colaboradores, ${ }^{24}$ que observaram o efeito de um programa de exercício com duração de 12 meses na qualidade de vida, aptidão aeróbia, rigidez arterial e função renal de pacientes com doença renal, no qual a maior parte do programa (11 meses) foi constituído por caminhada, realizada em ambiente domiciliar. Os indivíduos caminharam três vezes por semana a uma frequência cardíaca correspondente a 40-60\% do $\mathrm{VO}_{2}$ máx. A duração inicial das sessões variou de 5 a 20 minutos, de modo que o tempo de atividade aumentou de 5 a $10 \%$ semanalmente até 60 minutos. Apesar de não encontrarem melhoras na função renal em relação ao grupo-controle, diferenças significativas entre os grupos foram observadas para a qualidade de vida, aptidão aeróbia e rigidez arterial, reafirmando a importância da caminhada no controle da pressão arterial em pacientes renais pré-dialíticos.

Outra população que se beneficia acentuadamente dos programas de caminhada é a dos indivíduos com insuficiência cardíaca crônica (ICC). ${ }^{25,26}$ Nessa perspectiva, Fayazi e colaboradores $^{25}$ avaliaram a aptidão física de portadores de ICC antes e após 8 semanas de treinamento composto por caminhada, em 
ambiente domiciliar. Como resultados verificouse melhora significativa da distância percorrida no teste de 6 minutos, confirmando a melhora da capacidade funcional desses indivíduos.

Além da aptidão física, a caminhada promove melhoras em uma série de marcadores fisiológicos relacionados à ICC. Tsarouhas e colaboradores ${ }^{26}$ por exemplo, verificaram o efeito de 12 semanas de caminhada, com duração de 40 minutos, cinco vezes por semana, sobre a frequência cardíaca de recuperação, indicadores biológicos e a qualidade de vida de sujeitos com ICC. Quando comparados com o grupocontrole, os pacientes reduziram a frequência cardíaca pós-exercício e melhoraram a função endotelial. Esta evolução foi positivamente correlacionada com a distância percorrida em 40 minutos e a qualidade de vida também apresentou melhoras. Isso mostra que a prática regular de caminhada, mesmo no ambiente domiciliar, pode ser útil no tratamento dessa doença.

Como destacado, a prática da caminhada pode atuar tanto na redução da mortalidade por várias causas, como no aprimoramento da aptidão física e redução do uso de medicamentos em diferentes situações. Todavia, para que os efeitos positivos da caminhada nos indicadores de saúde sejam processados, algumas diretrizes quanto à prescrição do exercício devem ser consideradas. O tópico a seguir detalha algumas delas, destacando a aplicação de novos conceitos que podem ser usados para uma prescrição segura e eficiente do exercício.

\section{Considerações metodológicas na prescrição da caminhada}

Ao elaborar um programa de exercícios, os componentes essenciais de uma prescrição individualizada incluem seleção da modalidade, intensidade do esforço, duração, frequência semanal e progressão da atividade. Esses componentes são aplicados ao se desenvolver prescrições para pessoas de todas as idades e capacidades funcionais, independentemente da existência ou ausência de fatores de risco e doença. ${ }^{9}$ A tabela 1 apresenta diretrizes gerais de prescrição de exercícios, adaptadas para a prescrição da caminhada.

Contudo, o modo pelo qual cada componente é aplicado pode exercer um efeito diversificado no comportamento dos outros. Em que pese a importância de todos os componentes de prescrição na elaboração dos programas de atividades físicas, um dos aspectos mais difíceis de quantificar e controlar nas sessões de exercícios é a intensidade do esforço. Neste sentido, a seleção da atividade apropriada aparece como o primeiro item a ser definido, uma vez que traz consequências nas possibilidades de manutenção do esforço durante um tempo desejado. Entre as diversas atividades utilizadas no condicionamento aeróbio, a caminhada e a corrida são as mais populares, devido à facilidade para sua prática, além de constituírem formas de locomoção

Tabela 1. Considerações metodológicas para prescrição da caminhada em relação à duração, intensidade e frequência semanal.

\begin{tabular}{|c|c|c|c|c|c|c|c|}
\hline \multirow[b]{2}{*}{ Exercício } & \multirow[b]{2}{*}{ Duração (min) } & \multirow{2}{*}{$\begin{array}{c}\text { Classificação } \\
\text { do esforço }\end{array}$} & \multirow{2}{*}{$\begin{array}{c}\text { Frequência } \\
\text { Semanal }\end{array}$} & \multicolumn{4}{|c|}{ Intensidade do Exercício } \\
\hline & & & & \%FCmáx & $\% \mathrm{VO}_{2}$ máx & PSE & $\begin{array}{c}\%_{\mathrm{VO}_{2} \mathrm{R}} \\
\text { ou } \% \mathrm{FCR}\end{array}$ \\
\hline \multirow{3}{*}{ Caminhada } & $30-50$ & Leve & $3-6 x /$ sem & $57-63$ & $37-45$ & $9-11$ & $30-39$ \\
\hline & $30-40$ & Moderado & $3-6 x /$ sem & $64-76$ & $46-63$ & $12-13$ & $40-59$ \\
\hline & $20-30$ & Intenso & $3-6 x /$ sem & $77-95$ & $64-90$ & $14-17$ & $60-89$ \\
\hline
\end{tabular}

$\% \mathrm{VO}_{2} \mathrm{R}$ : percentual do consumo de oxigênio de reserva; \%FCmáx: percentual da frequência cardíaca máxima; \%FCR: percentual da frequência cardíaca de reserva; $\% \mathrm{VO}_{2}$ máx: percentual do consumo máximo de oxigênio; PSE: percepção subjetiva de esforço. 
mais usadas no cotidiano. Mas essas atividades apresentam aspectos biomecânicos e fisiológicos distintos, que podem influenciar sobremaneira na sustentação da atividade..$^{27,28}$ Nesse caso, optar pela caminhada ou pela corrida pode ter aplicações distintas na hora de prescrever o exercício aeróbio mais adequado.

Frequentemente nos deparamos com o seguinte questionamento: qual a melhor atividade, caminhada ou corrida? Quando o objetivo é locomover-se em baixas velocidades, a caminhada é certamente a atividade mais adequada. Em contraste, quando é desejado locomover-se em velocidades mais elevadas, a corrida deve ser selecionada. Obviamente, para os mal condicionados a caminhada deve ser a atividade preferida, passando para a corrida, à medida que a aptidão do praticante for melhorando.

Do ponto de vista estritamente físico, o trabalho para percorrer uma determinada distância independe do modo de locomoção (caminhar ou correr). Contudo, é possível que a demanda metabólica efetiva possa diferir na mesma velocidade entre essas duas modalidades de locomoção em função de diferenças na eficiência mecânica. Andar e correr são mais eficientes em velocidades, respectivamente, abaixo de $6 \mathrm{~km} \cdot \mathrm{h}^{-1} \mathrm{e}$ acima de $8 \mathrm{~km} \cdot \mathrm{h}^{-1}$, ${ }^{9}$ permanecendo, todavia, indefinida a melhor forma de locomoção na faixa intermediária de velocidade, ou seja, na intensidade de esforço a qual geralmente ocorre a transição entre a caminhada e a corrida. ${ }^{29}$ É nesta faixa de velocidade que as pessoas podem realizar tanto a caminhada quanto a corrida. É aí também que muitas dúvidas podem surgir acerca da melhor atividade para aprimorar o condicionamento aeróbio. Tal aspecto tornase ainda mais relevante, à medida que temos que elaborar programas de exercícios para indivíduos com problemas de saúde, por necessitarem de maiores cuidados no controle fisiológico do esforço.

Para tentar esclarecer esta questão, Monteiro e Araújo ${ }^{30}$ verificaram as respostas cardiorrespiratórias e perceptivas quando indivíduos caminharam e correram na velocidade de transição caminhada-corrida (VTCC), bem como em velocidades equivalentes a $\pm 0,5 \mathrm{~km} / \mathrm{h}$ desta. Os autores não observaram diferenças entre as variáveis aferidas, quando os participantes se exercitaram na VTCC, bem como abaixo desta. No entanto, quando os sujeitos caminharam a $0,5 \mathrm{~km} / \mathrm{h}$ acima da VTCC, a caminhada apresentou respostas cardiorrespiratórias e perceptivas significativamente superiores às verificadas na corrida. Outro aspecto a ser destacado é que os sujeitos relataram muitas dores nos membros inferiores. Levando em conta que o trabalho foi monitorado durante apenas oito minutos, isso leva a crer que dificilmente um praticante conseguiria passar de 10 minutos caminhando a uma velocidade $0,5 \mathrm{~km} / \mathrm{h}$ acima da velocidade de transição. Por isso, identificar a VTCC pode ser útil para verificar qual a velocidade máxima de caminhada que pode ser suportada em um programa de exercícios para indivíduos que realizam esta forma de locomoção.

Posteriormente, Farinatti e Monteiro ${ }^{31}$ compararam o comportamento das variáveis fisiológicas e de percepção ao esforço em indivíduos jovens e idosos, ao caminharem e correrem na VTCC. Foi possível observar que, para a população idosa, a corrida foi mais intensa do que a caminhada, devido às maiores respostas cardiorrespiratórias observadas, ao passo que para os jovens isso já não ocorreu. Portanto, na VTCC a prescrição da caminhada para a população idosa parece ser mais interessante, já que na corrida em apenas seis minutos de monitorização as respostas foram suficientes para elevar consideravelmente as variáveis metabólicas e perceptivas, relacionadas à fadiga e consequentemente à sustentação da atividade. Desta forma, a identificação da VTCC é crucial para prescrição de programas de caminhada em idosos.

Mais recentemente, Ivo ${ }^{32}$ também comparou as respostas cardiorrespiratórias e perceptivas na VTCC. Porém, diferentemente de Monteiro 
e colaboradores ${ }^{30}$ e Farinatti e Monteiro, ${ }^{31}$ que estudaram curtos períodos de tempo em situação de steady-state, esses autores simularam uma prescrição de 30 minutos de exercício. Em contraste com os estudos anteriores, o autor constatou um aumento das respostas cardiorrespiratórias, com vantagem para a corrida, a partir do décimo minuto de exercício. Se por um lado o maior gasto energético gerado pela corrida aponta para maiores benefícios à saúde, por outro o aumento de respostas, como frequência cardíaca e consumo de oxigênio, podem afetar a segurança e sustentação da atividade em indivíduos menos condicionados ou portadores de necessidades especiais. Por esse motivo a seleção da atividade ideal na VTCC dependerá do objetivo do programa de exercício, das características clínicas e do nível de aptidão física do praticante. Nesse sentido, indivíduos mal condicionados devem optar pela caminhada, ao passo que sujeitos com aptidão um pouco melhor e que já conseguem manter a corrida na VTCC por mais de 10 minutos devem utilizar a corrida como opção de exercício.

Como visto, a identificação da VTCC pode ser importante para determinar limites de velocidade para a caminhada em indivíduos mal condicionados, ou mesmo para determinar a velocidade inicial de corrida em praticantes que desejam iniciar a corrida. Contudo, a literatura tem destacado que a VTCC é protocolo-dependente, ou seja, a forma pela qual aumentamos a velocidade na esteira em dado protocolo, poderá determinar uma maior ou menor VTCC. ${ }^{33,34}$

Pensando nisso, Monteiro e Araújo ${ }^{30}$ determinaram a reprodutibilidade de um teste para detecção da VTCC em adultos jovens. O protocolo foi aplicado em três dias, separados por 24 a 48 horas, e iniciava com um aquecimento a $5 \mathrm{~km} / \mathrm{h}$ durante 2 minutos. Após esse período a velocidade era aumentada para $5,5 \mathrm{~km} / \mathrm{h}$ e, a cada 15 segundos, um incremento de $0,1 \mathrm{~km} / \mathrm{h}$ de velocidade era aplicado até o indivíduo atingir a VTCC. Cada indivíduo permaneceu por mais 1,5 minutos, após transitar da caminhada para a corrida. Este procedimento foi adotado para que o indivíduo pudesse certificar que aquela era realmente a velocidade de transição. Caso a velocidade não fosse confirmada, os sujeitos poderiam voltar a andar e o protocolo prosseguia até que a VTCC fosse alcançada por definitivo. É importante destacar que os sujeitos foram instruídos a modificar a forma de locomoção da caminhada para a corrida, quando não se sentissem mais confortáveis para caminhar. Ou seja, a VTCC seria a maior velocidade que poderia ser mantida de forma confortável, o que se diferencia da velocidade máxima de caminhada. Como resultados, variações mínimas foram verificadas nas velocidades de transição nos três dias de testes, tendo o protocolo apresentado um elevado coeficiente de relação intraclasse (ICC $=0,92)$. Além disso, quando as velocidades se diferenciaram entre as visitas, tal diferença não excedeu $0,2 \mathrm{~km} / \mathrm{h}$, comprovando que o protocolo é altamente reprodutível e pode ser utilizados na detecção da VTCC.

Posteriormente, Farinatti e Monteiro ${ }^{31}$ delinearam um protocolo semelhante ao supracitado. Nesse caso, apenas a velocidade de aquecimento e a progressão de cargas foram ligeiramente modificadas. Enquanto a velocidade do aquecimento foi de $4,5 \mathrm{~km} / \mathrm{h}$, o incremento de cargas em cada estágio de teste foi de $0,2 \mathrm{~km} / \mathrm{h}$. A reprodutibilidade do protocolo também foi testada em três dias, tanto em indivíduos jovens como em idosos. Não foram encontradas diferenças significativas entre as velocidades de transição detectadas nos três dias de teste e o coeficiente de correlação intraclasse também foi elevado, tanto em jovens $(\mathrm{ICC}=0,92)$ quanto em idosos $(\mathrm{ICC}=0,90)$. Como visto, os dois protocolos descritos podem ser úteis para detectar a VTCC, contudo, em virtude do segundo protocolo ter sido testado também em idosos e requerer um menor tempo de aplicação, este deve ser preferido.

Após detectar a VTCC, o passo seguinte consiste em utilizá-la na prescrição do exercício. Para tanto, sugerimos começar o treinamento 
caminhando na VTCC, durante 20 a 30 minutos. Quando o praticante for muito mal condicionado, sugere-se que a caminhada seja executada a $0,5 \mathrm{~km} / \mathrm{h}$ abaixo da VTCC. Quando o praticante conseguir caminhar 30 minutos na VTCC, deverá adotar a corrida na VTCC como forma de locomoção. Nesse caso, 1 a 2 minutos de corrida podem ser intercalados com caminhadas de 4 a 5 minutos, sempre na VTCC. Com a evolução do treinamento, o objetivo é aumentar o tempo de corrida em relação à caminhada, mantendo a VTCC como referência. A partir do momento em que o praticante conseguir correr durante 30 minutos na VTCC, a velocidade de corrida será então aumentada.

\section{Considerações finais}

A caminhada é um potencial instrumento na prevenção e tratamento de diversas doenças e fatores de risco associados. Além de não exigir técnica para sua realização - o que a torna atrativa para diferentes populações -, apresenta baixo risco de lesões e pode ser realizada em locais variados. Embora a prática da caminhada associada à redução de fatores de risco e prevenção de doenças careça de maiores esclarecimentos quanto à dose-resposta, sabe-se que atua positivamente nesses aspectos.

Contudo, para que os benefícios da caminhada sejam alcançados, as variáveis de prescrição do exercício, incluindo duração, intensidade do esforço e frequência semanal de treinamento, devem ser adequadamente quantificadas. É nesse sentido que a detecção da VTCC pode ser útil no delineamento das sessões de treinamento. Estudos têm demonstrado que caminhar em faixas de velocidade próximas da VTCC pode ser determinante para a segurança e a sustentação do exercício por períodos que tragam os benefícios desejados. Ademais, baseado nesta velocidade, é possível realizar uma prescrição progressiva e segura, de modo que o praticante possa evoluir da caminhada para a corrida, quando desejado. Logo, programas de exercícios para indivíduos mal condicionados ou com problemas de saúde, que buscam prevenção ou reabilitação de doenças, devem considerar esta alternativa em suas prescrições e exercícios.

\section{Referências}

1. World Health Organization. Global status report on noncommunicable diseases. Geneva: World Health Organization; 2011 Apr. 176 p.

2. Kohl HW, Craig CL, Lambert EV, Inoue S, Alkandari JR, Leetongin G, et al. The pandemic of physical inactivity: global action for public health. Lancet. $2012 \mathrm{Jul}$ 21;380(9838):294-305.

3. Murtagh EM, Murphy MH, Boone-Heinonen J. Walking - the first steps in cardiovascular disease prevention. Curr Opin Cardiol. 2010 Sep;25(5):490-6.

4. Fortes C, Mastroeni S, Sperati A, Pacifici R, Zuccaro P, Francesco F, et al. Walking four times weekly for at least $15 \mathrm{~min}$ is associated with longevity in a Cohort of very elderly people. Maturitas. 2012 Dec 29;S03785122(12):400-8.

5. Go AS, Mozaffarian D, Roger VL, Benjamim EJ, Berry JD, Borden WB, et al. Executive summary: heart disease and stroke statistics--2013 update: a report from the American Heart Association. Circulation. 2013 Jan 1;127(1):143-52.

6. Lee IM, Buchner DM. The importance of walking to public health. Med Sci Sports Exerc. 2008 Jul;40(7 Suppl):S512-8.

7. Colbert LH, Hootman JM, Macera CA. Physical activity - related injuries in walkers and runners in the aerobics center longitudinal study. Clin J Sport Med. 2000 Out;10(4):259-63.

8. Monteiro WD, Araújo CG. Cardiorespiratory and perceptual responses to walking and running at the same speed. Arq Bras Cardiol. 2009 Oct;93(4):418-25, 410-7.

9. American College of Sports Medicine. ACSM's Guidelines for Exercise Testing and Prescription. 8th ed. Philadelphia (PA): Lippincott Williams \& Wilkins; 2010. 400 p.

10. Garber CE, Blissmer B, Deschenes MR, Franklin BA, Lamonte MJ, Lee IM, et al. American College of Sports Medicine position stand. Quantity and quality of exercise for developing and maintaining cardiorespiratory, musculoskeletal, and neuromotor fitness in apparently healthy adults: guidance for prescribing exercise. Med Sci Sports Exerc. 2011 Jul;43(7):1334-59.

11. Landi F, Russo A, Cesari M, Pahor M, Liperoti R, Danese P, et al. Walking one hour 
or more per day prevented mortality among older persons: Results from ilSIRENTE study. Prev Med. 2008 Oct;47(4):422-6.

12. Nagai M, Kuriyama S, Kakizaki M, OhmoriMatsuda K, Sone T, Hozawa A, et al. Impact of walking on life expectancy and lifetime medical expenditure: the Ohsaki Cohort Study. BMJ Open. 2011 Jan 1;1(2):1-7.

13. Williams PT. Reduced diabetic, hypertensive, and cholesterol medication use with walking. Med Sci Sports Exerc. 2008 Mar;40(3):433-43.

14. Ministério da Saúde. Indicadores de saúde (IDS). Brasília. [acesso em 05 de março de 2013; atualizada em 2011]. Disponível em: http://tabnet.datasus.gov.br/cgi/deftohtm. exe?idb2011/c08.def.

15. Bond Brill J, Perry AC, Parker L, Robinson A, Burnett K. Dose-response effect of walking exercise on weight loss. How much is enough? Int J Obes Relat Metab Disord. 2002 Nov;26(11):1484-93.

16. Smith TC, Wingard DL, Smith B, KritzSilverstein D, Barrett-Connor E. Walking decreased risk of cardiovascular disease mortality in older adults with diabetes. J Clin Epidemiol. 2007 Mar;60(3):309-17.

17. Farinatti P, Oliveira RB, Pinto VLM, Monteiro WD, Francischetti E. Programa domiciliar de exercícios: efeitos de curto prazo sobre a aptidão física e pressão arterial de indivíduos hipertensos. Arq Bras Cardiol. 2005 Jun;84(6):473-9.

18. Liu S, Goodman J, Nolan R, Lacombe S, Thomas SG. Blood pressure responses to acute and chronic exercise are related in prehypertension. Med Sci Sports Exerc. 2012 Sep;44(9):1644-52.

19. Walker KZ, Piers LS, Putt RS, Jones JA, O'Dea K. Effects of regular walking on cardiovascular risk factors and body composition in normoglycemic women and women with type 2 diabetes. Diabetes Care. 1999 Apr;22(4):555-61.

20. Srinivasan SR, Wang R, Chen W, Wei CY, $\mathrm{Xu}$ J, Berenson GS. Utility of waist-to-height ratio in detecting central obesity and related adverse cardiovascular risk profile among normal weight younger adults (from the Bogalusa Heart Study). Am J Cardiol. 2009 Sep 1;104(5):721-4.

21. Figueroa A, Baynard T, Fernhall B, Carhart R, Kanaley JA. Endurance training improves post-exercise cardiac autonomic modulation in obese women with and without type 2 diabetes. Eur J Appl Physiol. 2007 Jul;100(4):437-44.

22. Saran AM, DuBose TD Jr. Cardiovascular disease in chronic kidney disease. Ther Adv Cardiovasc Dis. 2008;2(6):425-34.
23. Kosmadakis GC, John SG, Clapp EL, Viana JL, Smith AC, Bishop NC, et al. Benefits of regular walking exercise in advanced predialysis chronic kidney disease. Nephrol Dial Transplant. 2012 Mar;27(3):997-1004.

24. Mustata S, Groeneveld S, Davidson W, Ford G, Kiland K, Manns B. Effects of exercise training on physical impairment, arterial stiffness and health-related quality of life in patients with chronic kidney disease: a pilot study. Int Urol Nephrol. 2011 Dec;43(4):113341.

25. Fayazi S, Zarea K, Abbasi A, Ahmadi F. Effect of home-based walking on performance and quality of life in patients with heart failure. Scand J Caring Sci. 2013;27(2):246-52.

26. Tsarouhas K, Karatzaferi C, Tsitsimpikou C, Haliassos A, Kouretas D, Pavlidis P, et al. Effects of walking on heart rate recovery, endothelium modulators and quality of life in patients with heart failure. Eur J Cardiovasc Prev Rehabil. 2011 Aug;18(4):594-600.

27. Sasaki K, Neptune RR. Differences in muscle function during walking and running at the same speed. J Biomech. 2006;39(11):2005-13.

28. Mercier J, Le Gallais D, Durand M, Goudal C, Micallef JP, Préfant C. Energy expenditure and cardiorespiratory responses at the transition between walking and running. Eur J Appl Physiol Occup Physiol. 1994;69(6):525-9.

29. Monteiro WD, Araújo CG. Transição caminhada-corrida: considerações fisiológicas e perspectivas para estudos futuros. Rev Bras Med Esporte. 2001;7(6):207-22.

30. Monteiro WD, Araújo CG. Respostas cardiorrespiratórias e perceptivas para as mesmas velocidades de caminhada e corrida. Arq Bras Cardiol. 2009 Oct;93(4):418-25.

31. Farinatti PT, Monteiro WD. Walk-run transition in young and older adults: with special reference to the cardio-respiratory responses. Eur J Appl Physiol. 2010 Jun;109(3):379-88.

32. Ivo EX. Comparação das respostas cardiorrespiratórias e perceptivas na caminhada e corrida, quando realizadas na velocidade de transição caminhada-corrida [dissertação]. [Niterói]: Universidade Salgado de Oliveira; 2011.

33. Hreljac A, Imamura R, Escamilla RF, Edwards WB. Effects of changing protocol, grade, and direction on the preferred gait transition speed during human locomotion. Gait Posture. 2007 Mar;25(3):419-24.

34. Van Caekenberghe I, Segers V, De Smet K, Aerts P, De Clercq D. Influence of treadmill acceleration on actual walk-to-run transition. Gait Posture. 2010 Jan;31(1):52-6. 


\section{Autores}

\section{Ada Fernanda P. S. Lima}

Laboratório de Atividade Física e Promoção da Saúde. Instituto de Educação Física e Desportos. Universidade do Estado do Rio de Janeiro. Rio de Janeiro, RJ, Brasil.

\section{Alexandre H. Okano}

Departamento de Educação Física. Centro de Ciências da Saúde. Universidade Federal do Rio Grande do Norte. Natal, RN, Brasil.

\section{Alice R. Sampaio}

Laboratório de Atividade Física e Promoção da Saúde. Instituto de Educação Física e Desportos. Universidade do Estado do Rio de Janeiro. Rio de Janeiro, RJ, Brasil.

\section{Ana Paula M. Guttierres}

Laboratório de Atividade Física e Promoção da Saúde. Instituto de Educação Física e Desportos. Universidade do Estado do Rio de Janeiro. Rio de Janeiro, RJ, Brasil.

\section{Astrogildo V. Oliveira Júnior}

Departamento de Educação Física e Folclore. Colégio Pedro II. Rio de Janeiro, RJ, Brasil.

\section{Brenno S. Silva}

Programa de Pós-graduação em Ciências da Atividade Física. Universidade Salgado de Oliveira. Niterói, RJ, Brasil.

\section{Daniel A. Bottino}

Laboratório de Pesquisas Clínicas e Experimentais em Biologia Vascular. Centro Biomédico.

Universidade do Estado do Rio de Janeiro. Rio de Janeiro, RJ, Brasil.

\section{Dionizio Mendes Ramos Filho}

Laboratório de Bioenergética e Fisiologia Mitocondrial. Centro de Ciências da Saúde. Universidade Federal do Rio de Janeiro. Rio de Janeiro, RJ, Brasil.

\section{Elirez B. Silva}

Laboratório de Pesquisa Clínica Escola. Departamento de Fisioterapia. Universidade Gama Filho. Rio de Janeiro, RJ, Brasil.

\section{Ercole C. Rubini}

Laboratório de Fisiologia do Exercício. Universidade Estácio de Sá. Rio de Janeiro, RJ, Brasil.

\section{Fabrício V. A. Vasconcellos}

Centro de Investigação, Formação, Inovação, Intervenção e Desporto. Faculdade de Desporto. Universidade do Porto. Porto, Portugal.

\section{Felipe A. da Cunha}

Programa de Pós-graduação em Ciências Médicas. Universidade do Estado do Rio de Janeiro. Rio de Janeiro, RJ, Brasil.

\section{Fernanda Monteiro}

Laboratório de Atividade Física e Promoção da Saúde. Instituto de Educação Física e Desportos. Universidade do Estado do Rio de Janeiro. Rio de Janeiro, RJ, Brasil.

\section{Flávia Porto}

Programa de Pós-graduação em Ciências do Exercício e do Esporte. Universidade Gama Filho. Rio de Janeiro, RJ, Brasil. 


\section{Gustavo C. Lopes}

Laboratório de Atividade Física e Promoção da Saúde. Instituto de Educação Física e Desportos. Universidade do Estado do Rio de Janeiro. Rio de Janeiro, RJ, Brasil.

\section{Jonas L. Gurgel}

Programa de Pós-graduação em Ciências

Cardiovasculares. Universidade Federal Fluminense.

Niterói, RJ, Brasil.

\section{Jonathan Myers}

VA Palo Alto Health Care System. Cardiology Division. Stanford University. Palo Alto, California, United States.

\section{Karynne Grutter}

Laboratório de Anatomia Humana. Universidade Castelo Branco. Rio de Janeiro, RJ, Brasil.

\section{Lenifran Matos-Santos}

Programa de Pós-graduação em Ciências da Atividade Física. Universidade Salgado de Oliveira. Niterói, RJ, Brasil.

\section{Lorena Paes}

Programa de Pós-graduação em Fisiopatologia Clínica e Experimental. Universidade do Estado do Rio de Janeiro. Rio de Janeiro, RJ, Brasil.

\section{Luciane P. da Costa}

Laboratório de Atividade Física e Promoção da Saúde. Instituto de Educação Física e Desportos. Universidade do Estado do Rio de Janeiro. Rio de Janeiro, RJ, Brasil.

\section{Luiz G. Kraemer-Aguiar}

Departamento de Medicina Interna. Faculdade de Ciências Médicas. Universidade do Estado do Rio de Janeiro. Rio de Janeiro, RJ, Brasil.

\section{Matheus R. Hausen}

Laboratório de Atividade Física e Promoção da Saúde. Instituto de Educação Física e Desportos. Universidade do Estado do Rio de Janeiro. Rio de Janeiro, RJ, Brasil.

\section{Paulo T. V. Farinatti}

Laboratório de Atividade Física e Promoção da Saúde. Instituto de Educação Física e Desportos. Universidade do Estado do Rio de Janeiro. Rio de Janeiro, RJ, Brasil.

\section{Rafael A. Montenegro}

Programa de Pós-graduação em Fisiopatologia Clínica e Experimental. Universidade do Estado do Rio de Janeiro. Rio de Janeiro, RJ, Brasil.

\section{Raul A. Freire}

Programa de Pós-graduação em Ciências da Atividade Física. Universidade Salgado de Oliveira. Niterói, RJ, Brasil.

\section{Renato O. Massaferri}

Programa de Pós-graduação em Ciências da Atividade Física. Universidade Salgado de Oliveira. Niterói, RJ, Brasil.

\section{Ricardo B. Oliveira}

Laboratório de Atividade Física e Promoção da Saúde. Instituto de Educação Física e Desportos. Universidade do Estado do Rio de Janeiro. Rio de Janeiro, RJ, Brasil.

\section{Ricardo G. Cordeiro}

Programa de Pós-graduação em Ciências da Atividade Física. Universidade Salgado de Oliveira. Niterói, RJ, Brasil.

\section{Sérgio Machado}

Programa de Pós-graduação em Ciências da Atividade Física. Universidade Salgado de Oliveira. Niterói, RJ, Brasil.

\section{Taciana Pinto}

Laboratório de Atividade Física e Promoção da Saúde. Instituto de Educação Física e Desportos. Universidade do Estado do Rio de Janeiro. Rio de Janeiro, RJ, Brasil.

\section{Tainah P. Lima Monteiro}

Programa de Pós-graduação em Ciências Médicas. Faculdade de Ciências Médicas. Universidade do Estado do Rio de Janeiro. Rio de Janeiro, RJ, Brasil.

\section{Tânia M. P. F. Paschoalino}

Hospital Universitário Antônio Pedro. Universidade Federal Fluminense. Niterói, RJ, Brasil.

\section{Walace D. Monteiro}

Laboratório de Atividade Física e Promoção da Saúde. Instituto de Educação Física e Desportos. Universidade do Estado do Rio de Janeiro. Rio de Janeiro, RJ, Brasil. 